This is an author produced version of a paper published in Food Chem Toxicol. This paper has been peer-reviewed but does not include the final publisher proofcorrections or journal pagination.

Citation for the published paper:

Carnerup, Martin A and Saillenfait, Anne Marie and Jönsson, Bo A G "Concentrations of N-methyl-2-pyrrolidone (NMP) and its metabolites in plasma and urine following oral administration of NMP to rats."

Food Chem Toxicol. 2005 Sep;43(9):1441-7.

http://dx.doi.org/10.1016/j.fct.2005.04.007

Access to the published version may require journal subscription.

Published with permission from: Elsevier 


\section{Concentrations of $N$-methyl-2-pyrrolidone (NMP) and its metabolites in plasma and urine following oral administration of NMP to rats}

Martin A. Carnerup ${ }^{1}$, Ph.D., Anne Marie Saillenfait ${ }^{2}$, Ph.D., Bo A. G. Jönsson ${ }^{1}$, Ph.D.

${ }^{1}$ Department of Occupational and Environmental Medicine, Institute of Laboratory Medicine, Lund University Hospital, SE-221 85 Lund, Sweden

${ }^{2}$ Institut National de Recherche et de Securité, Avenue de Bourgogne, BP 27, 54501

Vandoeuvre, France

Keywords: Biological levels; Developmental toxicity; Metabolism, $N$-methyl-2pyrrolidone; Rat

Corresponding author:

Martin A. Carnerup

Department of Occupational and Environmental Medicine

Lund University Hospital

S-221 85 Lund

Sweden

Telephone: +46-46-173198

Fax: $\quad+46-46-143702$

E-mail: martin.carnerup@ymed.lu.se 


\begin{abstract}
The primary aims were to study the metabolism in rats and to determine the biological levels after one oral developmentally toxic dose of $N$-methyl-2-pyrrolidone (NMP), a widely used industrial chemical. Non-pregnant female Sprague-Dawley rats were given an oral single dose of either a non-toxic dose of $125 \mathrm{mg} \mathrm{NMP/kg} \mathrm{(group} \mathrm{1)} \mathrm{by} \mathrm{gavage} \mathrm{or}$ a developmentally toxic dose of $500 \mathrm{mg} / \mathrm{kg}$ (group 2). Blood plasma (7 rats per time point) and urine (10 rats per time point) were sampled up to $72 \mathrm{~h}$ after administration and analyzed using mass spectrometry. In both plasma and urine NMP, 5-hydroxy- $N$-methyl2-pyrrolidone (5-HNMP), $N$-methylsuccinimide and 2-hydroxy- $N$-methylsuccinimide (2HMSI) and 2-pyrrolidone (2-P) were identified. In urine $48 \%$ of the administered dose was recovered as 5-HNMP and 2-5\% as 2-HMSI. The total recovery in urine was 5359\%. The peak concentrations for NMP in plasma were 1.2 and $6.9 \mathrm{mmol} / \mathrm{l}, 0.42$ and $0.76 \mathrm{mmol} / \mathrm{l}$ for 5-HNMP, 0.07 and $0.31 \mathrm{mmol} / \mathrm{l}$ for MSI and for 2-HMSI the concentrations were 0.02 and $0.05 \mathrm{mmol} / \mathrm{l}$ for group 1 and 2, respectively. In summary, the same metabolites were found in rats as in humans and the biological levels were reported for NMP and its metabolites after oral exposure to a developmentally toxic dose and one non-toxic dose of NMP.
\end{abstract}




\section{Introduction}

$N$-methyl-2-pyrrolidone (NMP, CAS no. 872-50-4) is a small cyclic amide totally miscible in water as well as in most organic solvents. Due to its strong and selective power as a solvent, it is a widely used compound. E.g., NMP is used in the petrochemical industry, in the microelectronics fabrication industry and in the manufacturing of various compounds such as pigments, cosmetics, drugs and pesticides (International program on Chemical Safety, 2001). It is also used as a graffiti remover (Anundi et al., 1993 and 2000; Langworth et al., 2001) and has been investigated as a transdermal enhancer in the pharmaceutical industry (Barry and Bennett, 1987; Priborský et al., 1988).

In the rat, an extensive oral (Midgley et al., 1992) and percutaneous (Midgley et al., 1992; Payan et al., 2003) uptake has been shown. The metabolism is unclear but Wells et al. (1992) showed that the major urinary metabolite was 5-hydroxy- $N$-methyl-2pyrrolidone (5-HNMP).

In humans, NMP is easily absorbed in the airways (Åkesson and Paulsson, 1997), from the gastrointestinal tract (Åkesson and Jönsson, 1997) and through the skin (Ursin et al., 1995; Akrill et al., 2002; Åkesson et al., 2004). Furthermore, a human metabolic pathway have been described (figure 1), where NMP is hydroxylated to 5-HNMP, further oxidized to $\mathrm{N}$-methylsuccinimide (MSI) which is then hydroxylated to 2-hydroxy- $N$ methylsuccinimide (2-HMSI).

A human stillbirth after an occupational exposure, including skin contact, to an unknown level of NMP has been described in a case report (Solomon et al., 1996). Furthermore, in rats, NMP have been reported to be a developmentally toxic compound after dermal and oral administration (Becci et al., 1982; Hass et al., 1995; Saillenfait et 
al., 2002). Retarded growth has been reported after inhalation exposure (Saillenfait et al., 2003). It is unclear whether it is NMP itself or some of its metabolites that cause this effect.

Many fertile women are exposed in the industry and it is therefore important to develop good methods for exposure assessment of NMP. Several methods for biological monitoring of NMP exposure have been described where NMP and all of its identified metabolites have been suggested as biomarkers of exposure (NMP; Åkesson and Paulsson, 1997, 5-HNMP; Åkesson and Jönsson, 2000, MSI; Jönsson and Åkesson, 2001, 2-HMSI; Jönsson and Åkesson, 2003). For all of these compounds the relationship between air and biomarker levels in humans are known. Thus, it is possible to make comparisons between the biomarker levels and the existing occupational exposure limits in air. However, it would also be useful for risk assessments to know the levels of NMP and its metabolites in rats following a developmentally toxic dose of NMP.

In this work we study the metabolic pathway for NMP in rats. Furthermore, we determine the biological levels after one oral dose that was developmentally toxic, and one oral dose that was developmentally non-toxic. We also study the biological levels in rat urine after multiple administration of NMP.

\section{Material and Methods}

\subsection{Chemicals}

The NMP (99.5\%) used for the administration was purchased from Merck (Darmstadt, Germany). For the work-up and the analysis of plasma and urine, NMP was manufactured by Lab-Scan (Dublin, Ireland). MSI was from Aldrich (Gillingham, UK) 
and 2-HMSI was from Aldrich (Milwaukee, WI, USA) while 2-P was from Janssen Chimica (Geel, Belgium). 5-HNMP and the deuterium labelled compounds $\left[{ }^{2} \mathrm{H}_{4}\right]-5-$ HNMP, $\left[{ }^{2} \mathrm{H}_{4}\right]$-MSI and $\left[{ }^{2} \mathrm{H}_{3}\right]$-2-HMSI were synthesized by Synthelec (Lund, Sweden). Deuterium labeled NMP ([$\left.\left.{ }^{2} \mathrm{H}_{9}\right]-\mathrm{NMP}\right)$ was manufactured by Cambridge Isotope Laboratories (Andover, MA, USA). Methanol and ethyl acetate were from Lab-Scan (Dublin, Ireland), 99.5\% ethanol from Kemetyl AB (Haninge, Sweden) and glacial acetic acid from Merck (Darmstadt, Germany).

\subsection{Study design}

\subsubsection{Animals}

Female non-pregnant Sprague-Dawley rats (Iffa Credo, Saint Germain sur L'Arbresle, France) weighing 180-235g were used for all the studies. The animals were acclimatized to laboratory conditions for at least 5 days prior initiating the studies. The animal rooms were maintained at $21 \pm 2^{\circ} \mathrm{C}$ with a relative humidity of $45 \pm 5 \%$ and with a 12 -hr light/dark cycle. Commercial food pellets (UAR Alimentation, Villemoisson, France) and filtered tap water were available ad libitum.

\subsubsection{Single Oral Administration}

The metabolism and the biological levels in plasma and urine in rats were studied. The rats were given a single oral dose of NMP by gavage with distilled water as vehicle (5 $\mathrm{ml} / \mathrm{kg}$ ). For plasma sampling 42 rats were exposed to a non-toxic level of $125 \mathrm{mg} / \mathrm{kg}$ (group 1) and 42 rats were exposed to $500 \mathrm{mg} / \mathrm{kg}$ (group 2), which corresponds to a 
developmentally toxic level found by Saillenfait et al. (2002). Each animal was fasted 16 hr prior to dosing, and food was returned $4 \mathrm{hr}$ after dosing. Blood was collected at 1, 2, 4, 12, 24 and 48 h post-dose. At every time point 7 rats/group were killed, by bleeding the abdominal aorta under isoflurane anaesthesia. Blood was collected in heparinized tubes and plasma was immediately separated by centrifugation at $3500 \mathrm{rpm}$ for $10 \mathrm{~min}$. Samples were frozen $\left(-20^{\circ} \mathrm{C}\right)$ until analysis. For urine sampling, 10 rats were exposed to $125 \mathrm{mg} / \mathrm{kg}$ (group 1) and 10 rats were exposed to $500 \mathrm{mg} / \mathrm{kg}$ (group 2) under the same treatment conditions. After dosing, the animals were immediately placed in individual metabolism cages for the collection of the urine at $+4^{\circ} \mathrm{C}$. Urine was collected three times a day in six- or twelve-hour intervals during three days.

\subsubsection{Repeated oral administration}

The biological levels in urine were studied in rats given three repeated doses of NMP. The rats were given three oral doses of NMP in 24-h intervals by gavage with distilled water as vehicle. A dose of $125 \mathrm{mg} / \mathrm{kg}$ was given to 10 rats and another 10 rats were given a dose of $500 \mathrm{mg} / \mathrm{kg}$. Animals were fasted overnight only before the first treatment. Urine was collected as described above but three times a day in six-or twelve-hour intervals during five days. No plasma was collected.

\subsection{Analytical methods}

5-HNMP and 2-HMSI were analyzed according to the method presented by Carnerup et al. (2001). NMP, MSI and 2-pyrrolidone (2-P) were also analysed with this method. Deuterium-labelled internal standards were used for each corresponding compound 
except for 2-P for which $\left[{ }^{2} \mathrm{H}_{9}\right]-\mathrm{NMP}$ was used. Internal standards were added to diluted plasma or urine. NMP and the metabolites were then trapped on ENV+ solid phase extraction columns, eluted with an 8:2 ethyl acetate/ethanol mixture and evaporated until dryness. Acidic water was added and the samples were then analyzed by liquid chromatography coupled to a tandem mass spectrometer (LC-MS/MS). Some minor changes were made from the original method. A heated nebulizer in the positive ion mode was used as the ion source instead of ion spray and the samples were diluted ten times with water before work-up. The mass spectrometric parameters for the compounds are shown in table 1. Furthermore, the detection limits and the precisions for NMP and its metabolites are shown in table 2. The detection limit was calculated according to Miller and Miller (1993). Chromatograms for the two main metabolites, 5-HNMP and 2-HMSI, are shown in figure 2.

\subsection{Toxicokinetic calculation and statistics}

The area under the plasma concentration-time curve (AUC) was calculated using the trapezoidal rule (Rowland and Tozer, 1995). Since the rats were killed at every time point of plasma sampling, the mean plasma concentrations per time point were used for the estimation of the AUC. Comparisons between groups were performed using the MannWhitney U-test.

\section{Results}

In both plasma and urine from the rats exposed to a single oral dose NMP, 5-HNMP, MSI, 2-HMSI and 2-P were identified. The recoveries of NMP and its metabolites in 
urine from the rats are shown in table 3. Thus, the recoveries were 58\% (range 53-64\%) in group 1 and 52\% (range 36-62\%) in group 2. The area under the plasma concentrationtime curve (AUC) and the median peak concentrations in plasma and urine and the time to reach the peaks are shown in table 4 .

In the repeated administration study where doses of $125 \mathrm{mg} / \mathrm{kg}$ and $500 \mathrm{mg} / \mathrm{kg}$ over three consecutive days were given to the rats three major peaks were found for NMP and all metabolites. The total recoveries after five days are shown in table 3 and the urine concentrations are shown in table 5. The peak time and peak concentrations for the first administration were similar to those found after single dosing. The time to peak during the repeated administration did not vary significantly for any of the metabolites. There was no obvious accumulation for any of the compounds during the repeated administration. Urine concentration-time curves for 2-HMSI is shown in figure 3.

\section{Discussion}

The most important results were the identification of unchanged NMP and the metabolites MSI, 2-HMSI and 2-P in plasma and urine from NMP exposed rats. Furthermore, the levels of NMP and its metabolites were reported after one developmentally toxic dose and one non-toxic dose of NMP.

Previously, only 5-HNMP has been confirmed, using mass spectrometry, as a metabolite of NMP in rats (Wells et al., 1992). However, Payan et al. (2003) suggested MSI and 2-HMSI as NMP metabolites in rats given a single dose of radioactively labelled NMP intravenously, by comparing HPLC retention times. These metabolites were confirmed using mass spectrometry in this study. Furthermore, in another study 
Wells and Digenis (1988) administered radiolabelled ${ }^{3} \mathrm{H}$ - and ${ }^{14} \mathrm{C}$-isomers of NMP to rats and studied the excretion of the radioactivity in the urine. In one experiment ring labelled ${ }^{14} \mathrm{C}-\mathrm{NMP}$ was used and three peaks from radioactively labelled compounds were obtained following reversed phase liquid chromatography of the urine. Furthermore, administration of NMP labelled with tritium in the 4-position (same as the 2-position in 2-HMSI) together with methyl labelled ${ }^{14} \mathrm{C}$-NMP revealed the same peaks for ${ }^{14} \mathrm{C}$ but only the last of these were labelled for tritium. This peak corresponded to the main metabolite and was the one later identified as 5-HNMP (Wells et al., 1992). In separations of the metabolites found in this study using a similar separation as used by Well and Digenis (1988), only 2-HMSI had a shorter retention time than 5-HNMP while the retention times for NMP, MSI and 2-P were all longer. Thus, one of the two minor peaks in that study probably corresponds to 2-HMSI. This is in accordance with the loss of tritium from the 4-position. The metabolite corresponding to the other peak probably remains to be identified. The fact that we only found approximately 55\% of the administered dose of NMP in this study supports the presence of another metabolite. The loss of tritium may indicate a metabolism in the 4-position for this compound as well. Faeces and tissues were not examined in the present experiments and this could contribute to the low urinary recovery. However, in previous studies only $1-2 \%$ of the administered dose was recovered in faeces after both oral and dermal administration and only small amount were found in expired air and tissues (Wells and Digenis, 1988; Midgley et al., 1992).

2-P was identified as a novel NMP metabolite in our study. This metabolite is probably formed directly from NMP by demethylation but should probably be of minor 
importance since the plasma level was small compared to NMP and the other metabolites. It remains to establish if 2-P is also a metabolite of NMP in humans.

In this study $33-54 \%$ of the administered dose was excreted as 5-HNMP. This is in accordance with results previously reported by Payan et al. (2002). In that study five groups of rats were intravenously administered different doses of methyl labelled ${ }^{14} \mathrm{C}$ NMP and urinary elimination of 5-HNMP accounted for $42-55 \%$ of the administered doses. Also, Payan et al. reported that $4-10 \%$ of the administered dose was eliminated as NMP in the urine while Wells and Digenis (1988) found no urinary excretion of unchanged NMP. In our study, unchanged NMP was found in urine but only at low levels (1.2-3.7\%). Furthermore, in this study $99-100 \%$ of the eliminated amount (51-58\% of the dose) was found within 24 h of the administration. Payan et al. (2002) reported similar results, $85-98 \%$ of the radioactivity in urine. However, for the two highest administrations (100 and $500 \mathrm{mg} / \mathrm{kg}$ ) in that study, an increased amount was eliminated in urine post $24 \mathrm{~h}$, indicating that there was a delay in urinary elimination. A delay in time to reach the peak concentration was also found for 5-HNMP in plasma and Payan et al. suggested this to be a result of a saturable elimination process of NMP. The AUC for NMP in that study was also significantly higher for the two higher doses than for the lower. A delay in time to reach the maximal concentration for 5-HNMP, MSI and 2HMSI was also found in plasma in our study, after administration of the higher dose. High concentrations of NMP in plasma, and relatively higher AUC for NMP and MSI at the higher dose compared to the lower one was also found in our study.

Repeated dosing of chemicals are important for studies of toxicokinetics. In addition, repeated dosing of NMP or its metabolites during several days may be an 
interesting regime for studying the developmental toxicity of these compounds. Thus, we investigated whether this regime caused any accumulation of any of the metabolites in rats as has been found in humans. However, there were no obvious evidence for accumulation but since only urine was sampled in this study it is not possible to draw a certain conclusion. Still ,we think that the lack of tissue distributed radioactivity in other studies (Wells and Digenis, 1988; Midgley et al., 1992) together with the rapid elimination of radioactivity found by Payan et al. (2002) indicate no or only little accumulation. In humans, repeated exposure to NMP will result in an accumulation of at least 2-HMSI due to its long half-life (Jönsson and Åkesson, 2003). Thus, the elimination processes seem to be faster in rats than humans.

The same metabolites that previously have been identified in humans were also identified in rats. However, there were some notable differences. The mean urinary excretion of 5-HNMP and 2-HMSI in volunteers orally exposed to NMP corresponded to $44 \%$ and $20 \%$, respectively, of the administered dose. The mean total recovery was $65 \%$ (Åkesson and Jönsson, 1997). In rats, the relative excreted amount of 5-HNMP was higher than in humans while it was 4-9 times lower for 2-HMSI. The differences between rats and humans were also obvious from the plasma values. Thus, only small differences were found when comparing the AUC or the plasma concentrations of 2-HMSI from rats in this study with a previous study by Åkesson et al. (2004) where male and female volunteers were topically exposed to $300 \mathrm{mg}$ of NMP. Thus, in that paper, these women had a median AUC for 2-HMSI of $230 \mu \mathrm{mol} * \mathrm{~h} / \mathrm{l}$, while rats exposed to a dose corresponding to the NOAEL (125 mg/kg), showed a median AUC of only $250 \mu \mathrm{mol} * \mathrm{~h} / \mathrm{l}$. The corresponding values for the plasma concentrations were 5.4 and $20 \mu \mathrm{mol} / \mathrm{l}$, 
respectively. The small differences found may be of importance if it is proved that 2HMSI may induce developmentally toxic effects. After administration of the higher dose (500 mg/kg) in rats, corresponding to two times the LOAEL, the AUC and plasma concentrations were $780 \mu \mathrm{mol} * \mathrm{~h} / \mathrm{l}$ and $50 \mu \mathrm{mol} / \mathrm{l}$, respectively. Thus, the systemic dose of 2-HMSI in rats was only 3-9 times larger than in female volunteers exposed to a dose that corresponds to an 8-h exposure of approximately $10 \mathrm{mg} \mathrm{NMP} / \mathrm{m}^{3}$. On the other hand, if NMP itself is proved to be developmentally toxic, the dose in rats was 970-1200 times larger. In that case, occupational exposure of NMP should probably be of minor risk of inducing developmentally toxic effects. The high levels obtained for NMP and 5-HNMP may indicate that one of these compounds are responsible for the toxic effects but this remains to be determined. However, extrapolation of animal results to humans should be interpreted with caution. Possible differences between oral, dermal, and inhalation routes of exposure should be considered. The pregnant state itself could also result in changes, both in the routes of absorption but also more particularly in the rates of metabolism.

In conclusion, we have reported levels of NMP and its metabolites in rats orally exposed to one developmentally toxic dose and one non-toxic dose of NMP. However, to be able to interpret these levels in terms of risk it is essential to perform more studies, e.g., it is necessary to have knowledge about which metabolite of NMP that causes the developmental toxicity. Such studies are in progress.

\section{Acknowledgements}


This work was supported by Institut National de Recherche et de Securite in Vandoeuvre, France, the Swedish Council for Work Life Research, the Swedish Research Council and the Medical Faculty at Lund University in Lund, Sweden. 


\section{References}

Åkesson, B., Jönsson, B.A.G., 1997. Major metabolic pathway for $N$-methyl-2pyrrolidone in humans. Drug Metab Dispos 25, 267-269.

Åkesson, B., Paulsson, K., 1997. Experimental exposure of male volunteers to $N$-methyl2-pyrrolidone (NMP): acute effects and pharmacokinetics of NMP in plasma and urine. Occup Environ Med 54, 236-240.

Åkesson, B., Jönsson, B.A.G., 2000. Biological monitoring of $N$-methyl-2-pyrrolidone using 5-hydroxy- $N$-methyl-2-pyrrolidone in plasma and urine as the biomarker. Scand $\mathrm{J}$ Work Environ Health 26, 213-218.

Åkesson, B., Carnerup, M.A., Jönsson, B.A.G., 2004. Evaluation of biomarkers of exposure by studying percutaneous absorption of $N$-methyl-2-pyrrolidone in volunteers. Scand J Work Environ Health. In Press.

Akrill, P., Cocker, J., Dixon, S., 2002. Dermal exposure to aqueous solutions of $\mathrm{N}$ methylpyrrolidone. Toxicol Lett 134, 265-269.

Anundi, H., Lind, M.L., Friis, L., Itkes, N., Langworth, S., Edling, C., 1993. High exposures to organic solvents among graffiti removers. Int Arch Occup Environ Health. 65, 247-251. 
Anundi, H., Langworth, S., Johanson, G., Lind, M-L., Åkesson, B., Friis, L., Itkes, N., Söderman, E., Jönsson, B.A.G., Edling, C., 2000. Air and biological monitoring of solvent exposure during graffiti removal. Int Arch Occup Environ Health 73, 561-569.

Barry, B.W., Bennett, S.L., 1987. Effect of penetration enhancers on the permeation of mannitol, hydrocortisone and progesterone through human skin. J Pharm Pharmacol 39, 535-546.

Becci, P.J., Knickerbocker, M.J., Raegan, E.L., Parent, R.A., Burnette, L.W., 1982. Teratogenicity study of $N$-methylpyrrolidone after dermal application to Sprague-Dawley rats. Fundam Appl Toxicol 2, 73-76.

Carnerup, M.A., Åkesson, B., Jönsson, B.A.G., 2001. Determination of 5-hydroxy- $N$ methyl-2-pyrrolidone and 2-hydroxy- $N$-methylsuccinimide in human plasma and urine using liquid chromatography-electrospray tandem mass spectrometry. J Chromatogr B $761,107-113$

Hass, U., Jakobsen, B.M., Lund, S.P., 1995. Developmental toxicity of inhaled $N$ methylpyrrolidone in the rat. Pharmacol Toxicol 76, 406-409.

International program on Chemical Safety (IPCS) (2001) No. 35. N-methyl-2pyrrolidone. Geneva, World Health Organization. 
Jönsson, B.A.G., Åkesson, B., 2001. N-Methylsuccinimide in plasma and urine as a biomarker of exposure to $N$-methyl-2-pyrrolidone. Int Arch Occup Environ Health 74, 289-294.

Jönsson, B.A.G., Åkesson, B., 2003. Human experimental exposure to $N$-methyl-2pyrrolidone (NMP): toxikokinetics of NMP, 5-hydroxy- $N$-methyl-2-pyrrolidone, $N$ methylsuccinimide and 2-hydroxy- $N$-methylsuccinimide (2-HMSI), and biological monitoring using 2-HMSI as a biomarker. Int Arch Environ Health 76, 267-274.

Langworth, S., Anundi, H., Friis, L., Johanson, G., Lind, M-L., Söderman, E., Åkesson, B.A., 2001. Acute health effects common during graffiti removal. Int Arch Occup Environ Health 74, 213-218.

Midgley, I., Hood, A.J., Chasseaud, L.F., Brindley, C.J., Baughman, S., Allan, G., 1992. Percutaneous absorption of co-administered $N$-methyl-2- $\left[{ }^{14} \mathrm{C}\right]$ pyrrolidinone and 2$\left[{ }^{14} \mathrm{C}\right]$ pyrrolidinone in the rat. Fd Chem Tixic 30, 57-64.

Miller, J.C., Miller, J.N., 1993. Statistics for Analytical Chemistry. Ellis Horwood, Chichester, pp. 115-117. 
Payan, J.P., Beydon, D., Fabry, J.P., Boudry, I., Cossec, B., Ferrari, E., 2002. Toxicokinetics and metabolism of $N-\left[{ }^{14} \mathrm{C}\right]$ methylpyrrolidone in male Sprague-Dawley rats. A saturable NMP elimination process. Drug Metab Dispos 30, 1418-1424.

Payan, J.P., Boudry, I., Beydon, D., Fabry, J.P., Grandclaude, M.C., Ferrari, E., Andre, J.C., 2003. Toxicokinetics and metabolism of $N$-[ $\left.{ }^{14} \mathrm{C}\right] N$-methyl-2-pyrrolidone in male Sprague-Dawley rats: in vivo and in vitro percutaneous absorption. Drug Metab Dispos 31, 659-669.

Priborský, J., Takayama, K., Nagai, T., Waitzova, D., Elis, J., Makino, Y., Suzuki, Y., 1988. Comparison of Penetration-enhancing ability of laurocapram, $N$-methyl-2pyrrolidone and dodecyl-L-pyroglutamate. Pharm Weekbl Sci 10, 189-192.

Rowland, M., Tozer, T.N., 1995. Clinical pharmacokinetics: concepts and applications 3rd ed. Lippincott Williams \& Wilkins, Philadelphia, PA, pp. 469-472.

Saillenfait, A.M., Gallissot, F., Langonné, I., Sabaté, J.P., 2002. Developmental toxicity of $N$-methyl-2-pyrrolidone administered orally to rats. Food Chem Toxicol 40, 17051712.

Saillenfait, A.M., Gallisso, F., Morel, G., 2003. Developmental toxicity of N-methyl-2pyrrolidone in rats following inhalation exposure. Food Chem Toxicol 41, 583-588. 
Solomon, G.M., Morse, E.P., Garbo, M.J., Milton, D.K., 1996. Stillbirth after occupational exposure to $N$-methyl-2-pyrrolidone. J Occup Environ Med 38, 705-713.

Ursin, C., Hansen, C.M., Van Dyk, J.M., Jensen, P.O., Christensen, I.J., Ebbehoej, J., 1995. Permeability of commercial solvents through living human skin. Am Ind Hyg Assoc J 56, 651-660.

Wells, D.A., Digenis, G.A., 1988. Disposition and metabolism of double-labeled $\left[{ }^{3} \mathrm{H}\right.$ and $\left.{ }^{14} \mathrm{C}\right] \mathrm{N}$-methyl-2-pyrrolidinone in the rat. Drug Metab Dispos 16, 243-249.

Wells, D.A., Hawi, A.A., Digenis, G.A., 1992. Isolation and Identification of the major urinary metabolite of $N$-methylpyrrolidinone in the rat. Drug Metab Dispos 20, 124-126. 


\section{Footnotes}

Abbreviations:

2-HMSI 2-hydroxy- $N$-methylsuccinimide, page 1 (title page)

5-HNMP 5-hydroxy- $N$-methyl-2-pyrrolidone, page 1 (title page)

2-P 2-pyrrolidone, page 1 (title page)

AUC area under the curve, page 1 (title page)

CYP2E1 cytochrome P450 isoform 2E1, page 1 (title page)

MSI methylsuccinimide, page 1 (title page)

NMP $\quad N$-methyl-2-pyrrolidone, page 1 (title page) 
Table 1: Mass spectrometric parameters for NMP, its metabolites and corresponding deuterium labeled internal standard.

\begin{tabular}{lllll}
\hline Compound & Precursor & Fragment & Declustering & Collision \\
& ion & ion & Potential & Energy \\
& $\mathbf{m} / \mathbf{z}$ & $\mathbf{m} / \mathbf{z}$ & $\mathbf{( V )}$ & $\mathbf{( V )}$ \\
\hline NMP & 99.8 & 58.3 & 30 & 31 \\
{$\left[{ }^{2} \mathrm{H}_{9}\right]-\mathrm{NMP}$} & 108.7 & 62.3 & 31 & 37 \\
5 5-HNMP & 115.8 & 57.2 & 30 & 31 \\
{$\left[{ }^{2} \mathrm{H}_{4}\right]-5-\mathrm{HNMP}$} & 119.8 & 60.5 & 30 & 31 \\
$\mathrm{MSI}$ & 114.1 & 58.2 & 30 & 21 \\
{$\left[{ }^{2} \mathrm{H}_{3}\right]-\mathrm{MSI}$} & 117.3 & 59.3 & 30 & 26 \\
$2-\mathrm{HMSI}$ & 129.9 & 58.3 & 30 & 31 \\
{$\left[{ }^{2} \mathrm{H}_{3}\right]-2-\mathrm{HMSI}$} & 132.9 & 61.2 & 30 & 31 \\
$2-\mathrm{P}$ & 85.7 & 44.2 & 26 & 35 \\
\hline
\end{tabular}


Table 2: Detection limits and precision for NMP and its metabolites.

\begin{tabular}{lllllll}
\hline Substance & \multicolumn{2}{l}{ Detection Limit $(\mathbf{n g} / \mathbf{m l})$} & & \multicolumn{2}{l}{ Precision $(\% \mathbf{C V})$} & \multicolumn{2}{c}{ Spiked conc. } \\
\cline { 2 - 3 } & Plasma & Urine & & Plasma & Urine & $(\mu \mathbf{g} / \mathbf{m l})$ \\
\hline NMP & 0.99 & 0.64 & 5 & 4 & 6.3 \\
5-HNMP & 93 & 72 & 5 & 7 & 6.3 \\
MSI & 110 & 11 & 4 & 5 & 6.3 \\
2-HMSI & 2.9 & 0.15 & 4 & 6 & 6.3 \\
2-P & 15 & 18 & 4 & 1 & 5.0 \\
\hline
\end{tabular}


Table 3: Median (range) recoveries of the administered dose for NMP and its metabolites after a single dose and after repeated doses on three consecutive days.

\begin{tabular}{cccc}
\hline Dose (mg/kg) & Substance & $\begin{array}{c}\text { Recovery single dose } \\
\text { Recovery repeated doses }\end{array}$ & $\mathbf{( \% )}$ \\
\hline 125 & NMP & $1.6(1.2-3.2)$ & $2.9(1.6-5.1)$ \\
125 & 5 -HNMP & $48(46-56)$ & $58(42-68)$ \\
125 & MSI & $0.1(0.1-0.1)$ & $1.1(0.8-1.4)$ \\
125 & $2-H M S I$ & $5.1(3.4-8.1)$ & $4.2(2.2-7.7)$ \\
125 & $2-P$ & $0.7(0.7-0.9)$ & $3.6(2.6-7.5)$ \\
500 & NMP & $2.6(1.2-3.7)$ & $4.6(2.1-6.1)$ \\
500 & $5-H N M P$ & $48(33-54)$ & $48(39-82)$ \\
500 & MSI & $0.1(0.1-0.3)$ & $1.1(0.6-3.1)$ \\
500 & $2-H M S I$ & $2.3(1.3-2.9)$ & $1.5(0.7-2.3)$ \\
500 & $2-P$ & $0.8(0.6-1.0)$ & $0.5(0.3-4.9)$ \\
\hline
\end{tabular}


Table 4: Median peak concentrations (range), time to reach the peak and median AUC

after a single oral dose of NMP.

\begin{tabular}{|c|c|c|c|c|c|c|c|}
\hline \multirow[t]{2}{*}{ Compound } & \multirow[t]{2}{*}{ Matrix } & \multicolumn{3}{|l|}{ Group 1} & \multicolumn{3}{|l|}{ Group 2} \\
\hline & & $\begin{array}{l}\text { Concentration } \\
(\mathrm{mmol} / \mathrm{l})\end{array}$ & $\begin{array}{l}\text { Time } \\
\text { (h) }\end{array}$ & $\begin{array}{l}\text { AUC } \\
\left(\mathrm{mmol}^{*} \mathrm{~h} / \mathrm{l}\right)\end{array}$ & $\begin{array}{l}\text { Concentration } \\
(\mathrm{mmol} / \mathrm{l})\end{array}$ & $\begin{array}{l}\text { Time } \\
\text { (h) }\end{array}$ & $\begin{array}{l}\text { AUC } \\
\left(\mathrm{mmol}^{*} \mathrm{~h} / \mathrm{l}\right)\end{array}$ \\
\hline NMP & Plasma & $1.2(0.95-1.4)$ & 1 & 7.1 & $6.9(0.36-7.2)$ & 2 & 58 \\
\hline 5-HNMP & Plasma & $0.42(0.36-0.60)$ & 4 & 4.3 & $0.76(0.57-1.1)$ & 12 & 13 \\
\hline MSI & Plasma & $0.07(0.06-0.10)$ & 4 & 0.46 & $0.31(0.15-0.44)$ & 12 & 3.9 \\
\hline 2-HMSI & Plasma & $0.02(0.02-0.08)$ & 12 & 0.30 & $0.05(0.02-0.14)$ & 12 & 0.65 \\
\hline $2-\mathrm{P}$ & Plasma & $0.01(0.01-0.01)$ & 4 & 0.10 & $0.04(0.03-0.04)$ & 4 & 0.60 \\
\hline NMP & Urine & $1.5(0.77-1.8)$ & $0-6$ & $\mathrm{NA}^{1}$ & $3.6(2.7-7.8)$ & $6-12$ & NA \\
\hline 5-HNMP & Urine & $41(40-58)$ & $6-12$ & NA & $96(86-113)$ & $6-12$ & NA \\
\hline MSI & Urine & $0.14(0.08-0.17)$ & $6-12$ & NA & $0.37(0.16-0.44)$ & $12-24$ & NA \\
\hline 2-HMSI & Urine & $3.5(2.0-8.8)$ & $6-12$ & NA & 4.4 (3.4-8.9) & $12-24$ & NA \\
\hline 2-P & Urine & $0.65(0.57-1.0)$ & $6-12$ & NA & $1.5(1.5-2.0)$ & $6-12$ & NA \\
\hline
\end{tabular}

${ }^{1}$ Not applicable 
Table 5: Median (range) peak concentrations ${ }^{1}$ after three administrations in $24 \mathrm{~h}$ intervals.

\begin{tabular}{lllll}
\hline Dose $(\mathbf{m g} / \mathbf{k g})$ & $\mathbf{2}$ Substance & Peak 1 & Peak 2 & Peak 3 \\
\hline 125 & NMP & $1.6(0.0-2.4)$ & $1.9(1.6-2.5)$ & $2.0(1.6-3.0)$ \\
125 & 5-HNMP & $39(31-69)$ & $33(12-46)$ & $35(13-58)$ \\
125 & MSI & $0.6(0.4-0.7)$ & $0.7(0.5-1.2)$ & $0.9(0.4-2.1)$ \\
125 & 2-HMSI & $2.2(0.6-4.1)$ & $1.6(0.5-3.0)$ & $2.8(1.6-6.3)$ \\
125 & 2-P & $2.8(1.8-5.1)$ & $2.1(0.6-3.2)$ & $2.5(0.1-4.3)$ \\
500 & NMP & $12(9.8-99)$ & $12(11-17)$ & $14(9.5-19)$ \\
500 & 5-HNMP & $111(70-163)$ & $129(86-177)$ & $166(130-236)$ \\
500 & MSI & $1.5(0.8-6.5)$ & $3.5(1.3-14)$ & $4.3(2.0-17)$ \\
500 & 2-HMSI & $3.0(1.2-7.1)$ & $2.9(1.5-6.0)$ & $6.0(3.8-9.7)$ \\
500 & 2-P & $1.0(0.9-6.1)$ & $4.3(1.8-5.0)$ & $2.2(1.9-2.4)$ \\
\hline
\end{tabular}

${ }^{1}$ Unit: $\mathrm{mmol} / \mathrm{l}$ 
Figure 1. The proposed metabolism of NMP in humans.

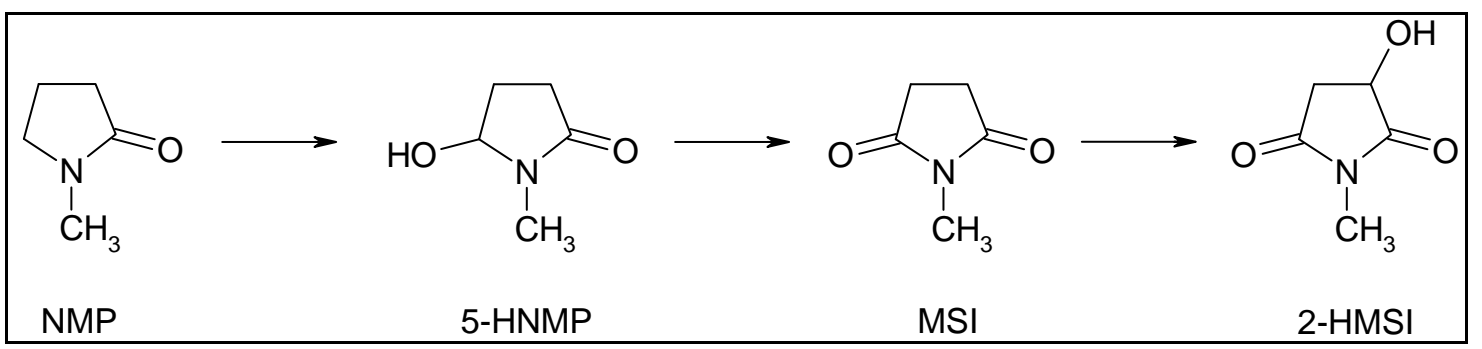


Figure 2. The chromatograms for the main metabolites, 5-HNMP (a) and 2-HMSI (b) in rat urine spiked to concentration of $1.0 \mu \mathrm{g} / \mathrm{ml}$.
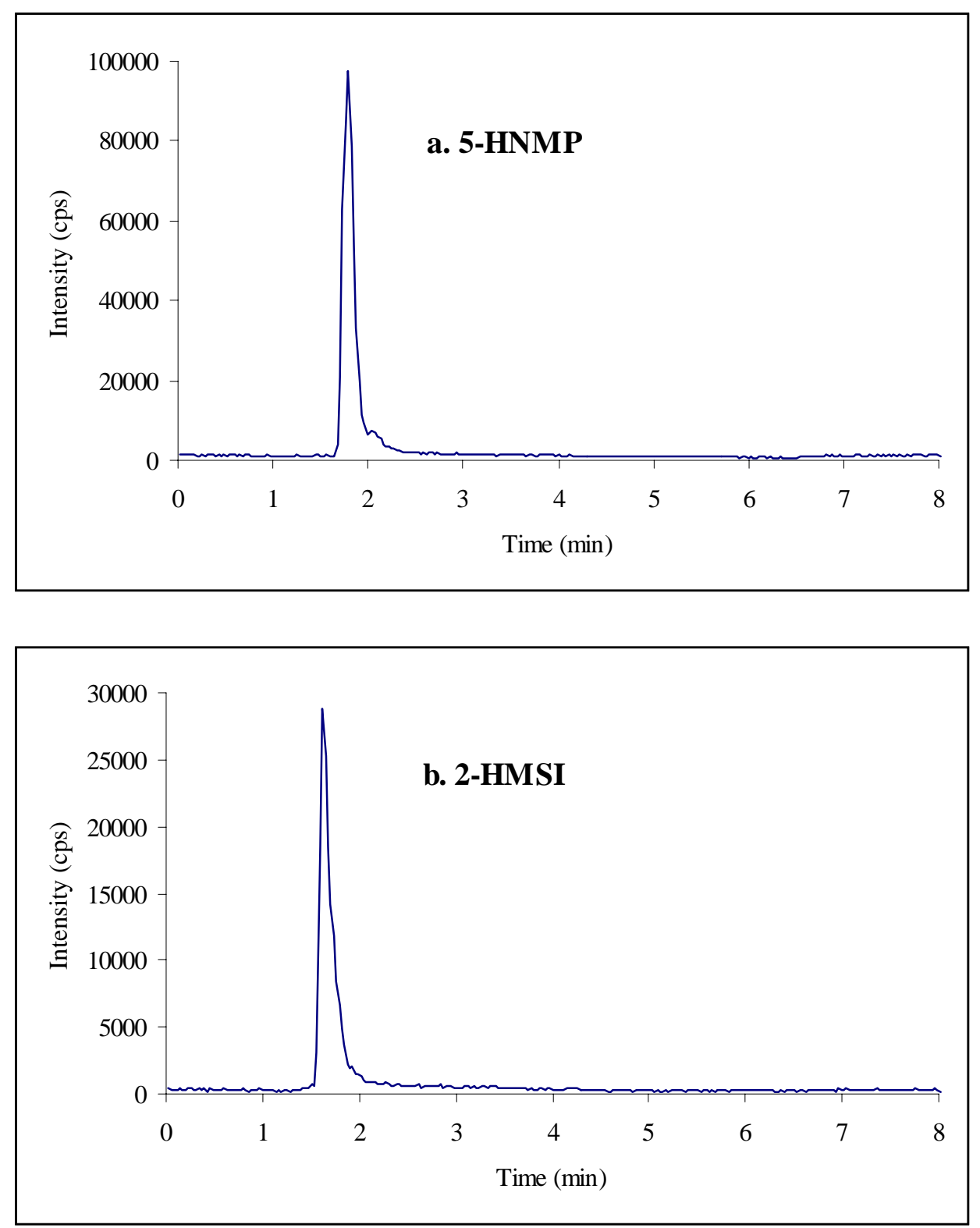
Figure 3. The urine concentration vs. time for 2-HMSI in rats given $125 \mathrm{mg} / \mathrm{kg}(\diamond)$ and $500 \mathrm{mg}$ NMP/kg ( $(\mathbf{})$ by gavage at 0,24 and $48 \mathrm{~h}$.

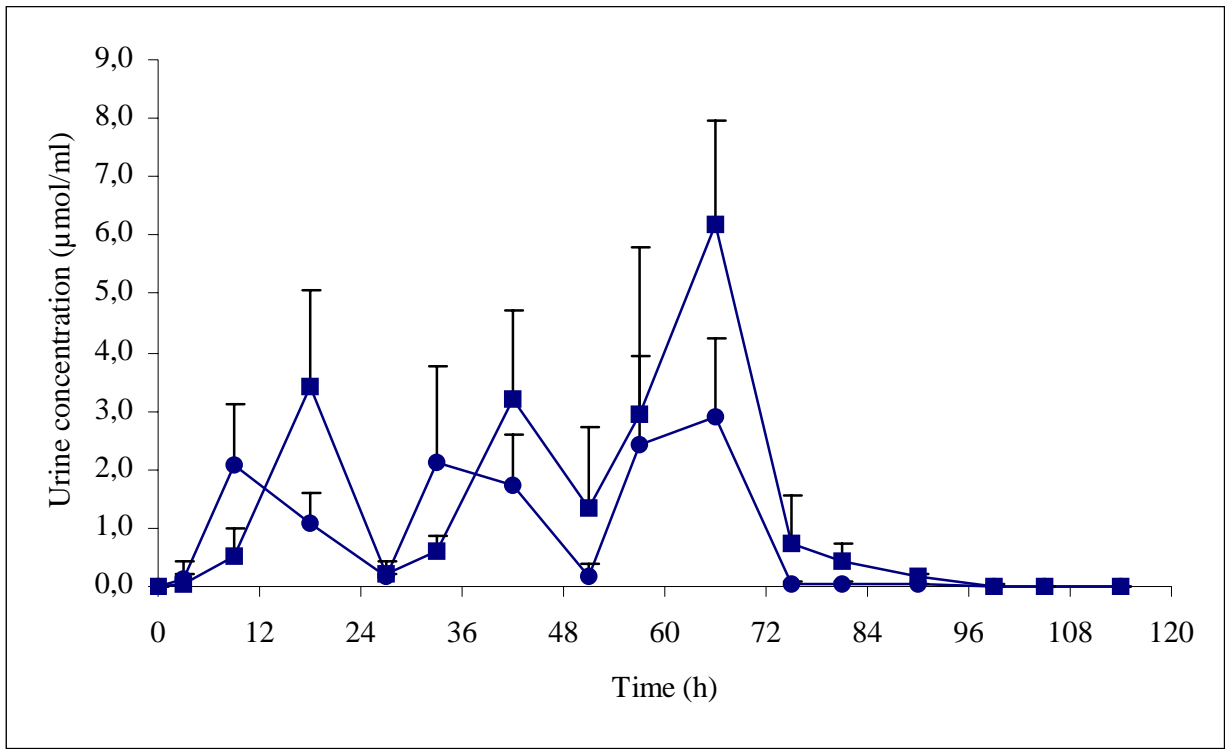

